Y. SHIBUYA

KODAI MATH. J.

5 (1982), 1-12

\title{
SOME ISOSPECTRAL PROBLEMS
}

\author{
By YhujI SHIBUya
}

\section{$\S 0$. Introduction}

In the previous paper [7], we proved the following theorem.

THEOREM 0.1. Let $M^{2 n+1}(c)$ be a compact Sasakian space from of a constant $\phi$-sectional curvature $c$ of dimension $2 n+1=5,7,9,11$ or 13 , and let $M^{*}$ be a compact Sasakian manifold. If $M^{2 n+1}(c)$ and $M^{*}$ are isospectral with respect to the Laplace operator, then $M^{*}$ is a $2 n+1$ dimensional Sasakian space form of a constant $\phi$-sectional curvature $c^{*}=c . \quad(c \neq 31$ when $2 n+1=13)$

In this paper, we study isospectral problems not only of Sasakian manifolds but of their submanifolds. In addition, by using the similar methods, we study isospectral problems of quaternion Kaehler manifolds and some submanifolds of Kaehler manifolds.

\section{$\S 1$. Special Classes of Sasakian Manifolds}

Let $M^{2 n+1}(c)$ be a $2 n+1(\geqq 5)$ dimensional Sasakian manifold with structure tensors $(\phi, \xi, \eta)$. In [7] we proved the following two propositions, which give the characterizations of Sasakian space forms and C-Einstern manifolds in terms of equalities involving curvature tensors.

Proposition 1.1. A Sasakıan manifold $M^{2 n+1}$ satısfies an ınequality

$$
|R|^{2} \geqq \frac{2}{n(n+1)} S^{2}-\frac{4(3 n+1)}{n+1} S+\frac{4 n(3 n+1)(2 n+1)}{n+1},
$$

where $S$ is the scalar curvature of $M^{2 n+1}$. Equality holds if and only if $M^{2 n+1}$ is a Sasakian space form.

Proposition 1.2. A Sasakıan manifold $M^{2 n+1}$ satısfies an inequality

$$
\mid \text { Ricci }\left.\right|^{2} \geqq \frac{(S-2 n)^{2}}{2 n}+4 n^{2} .
$$

Equality holds of and only if $M^{2 n+1}$ is a C-Einstern manfold.

See $[7]$ for notations and definitıons of Sasakıan manifolds.

Received March 13, 1980. 
We defined two more classes of Sasakian manifolds in [7]. A Sasakian manifold $M^{2 n+1}$ is said to have the $\eta$-parallel Ricci tensor if the Ricci tensor of $M^{2 n+1}$ satisfies

$$
\left(\nabla_{V} \operatorname{Ricci}\right)(\phi X, \phi Y)=0
$$

for any vector fields $X, Y$ and $V$ on $M^{2 n+1}$. And a Sasakian manifold $M^{2 n+1}$ is called a locally D-symmetric space if the curvature tensor of $M^{2 n+1}$ satisfies

$$
\left(\nabla_{V} R\right)(\phi X, \phi Y, \phi Z, \phi W)=0
$$

for any vector fields $X, Y, Z, V$ and $W$ on $M^{2 n+1}$.

The geometric meaning of the $\eta$-parallel Ricci tensor was explained from the view point of fibering. (See also Kon [4], Shibuya [8]) By using the similar point of view, we know the geometric meaning of locally $D$-symmetric spaces. (We have had the notion of locally $D$-symmetric spaces for a long time. Independently Takahashi studied the same notion, which he called locally $\phi$-symmetric spaces, and made same qualitative observations in his paper [9].)

In this section we establish two inequalities on Sasakian manifolds, which give the characterizations of Sasakian manifolds with $\eta$-parallel Ricci tensors and locally $D$-symmetric spaces respectively.

By using the curvature properties of Sasakian manifolds, we have

and

$$
\begin{aligned}
& \left(\nabla_{V} \operatorname{Ricci}\right)(\phi X, \phi Y) \\
=\left(\nabla_{V} \operatorname{Ricci}\right)(X, Y) & -2 n \eta(X) g(\phi V, Y)+\eta(X) \operatorname{Ricci}(\phi V, Y) \\
& -2 n \eta(Y) g(\phi V, X)+\eta(Y) \operatorname{Ricci}(\phi V, X)
\end{aligned}
$$

$$
\begin{aligned}
& \left(\nabla_{V} R\right)(\phi X, \phi Y, \phi Z, \phi W) \\
= & \left(\nabla_{V} R\right)(X, Y, Z, W) \\
& -\eta(X)(R(Z, W, V, \phi Y)+\Phi(Z, Y) g(W, V)-\Phi(W, Y) g(Z, V)) \\
& +\eta(Y)(R(Z, W, V, X)+\Phi(Z, X) g(W, V)-\Phi(W, X) g(Z, V)) \\
& -\eta(Z)(R(Z, Y, V, W)+\Phi(X, W) g(Y, V)-\Phi(Y, W) g(X, V)) \\
& +\eta(W)(R(X, Y, V, Z)+\Phi(X, Z) g(Y, V)-\Phi(Y, Z) g(X, V))
\end{aligned}
$$

for any vector fields $X, Y, Z, V$ and $W$ on $M^{2 n+1}$.

Taking the squares of the lengths of both sides of (1.5) and (1.6), we have

$$
\mid \nabla \text { Ricci }\left.\right|^{2}=2 \mid \text { Ricci }\left.\right|^{2}-8 n S+16 n^{3}+8 n^{2}
$$

and

$$
+\left(\text { length of }\left(\nabla_{V} \operatorname{Ricci}\right)(\phi X, \phi Y)\right)^{2}
$$




$$
\begin{aligned}
|\nabla R|^{2}= & 4|R|^{2}-16 S+32 n^{2}+16 n \\
& +\left(\text { length of }\left(\nabla_{V} R\right)(\phi X, \phi Y, \phi Z, \phi W)\right)^{2} .
\end{aligned}
$$

Thus we have the following two propositions.

Proposition 1.3. A Sasakıan manıfold $M^{2 n+1}$ satısfies an ınequality

$$
\mid \nabla \text { Ricci }\left.\right|^{2} \geqq 2 \mid \text { Ricci }\left.\right|^{2}-8 n S+16 n^{3}+8 n^{2} .
$$

Equality holds if and only if $M^{2 n+1}$ has the $\eta$-parallel Ruccl tensor.

Proposition 1.4. A Sasakıan manifold $M^{2 n+1}$ satısfies an ınequality

$$
|\nabla R|^{2} \geqq 4|R|^{2}-16 S+32 n^{2}+16 n .
$$

Equality holds of and only of $M^{2 n+1}$ is a locally D-symmetruc space.

As an application of (1.9), we give an alternate proof of a theorem of Okumura $[5]$.

COROLlaRy 1.5. A Sasakıan manifold $M^{2 n+1}$ with parallel Riccr tensor is an Eınstein manifold.

Proof. We first note that on a Riemannian manifold of dimension $2 n+1$ we have

$$
|\operatorname{Ricci}|^{2} \geqq \frac{1}{2 n+1} S^{2},
$$

where equality holds if and only if the Riemannian manifold is an Einstein manifold. Combining the assumption, (1.9) and (1.11), we have

$$
\begin{aligned}
\mid \text { Ricci }\left.\right|^{2} & =4 n S-8 n^{3}-4 n^{2} \\
& =\frac{2 S\left(4 n^{2}+2 n\right)-\left(4 n^{2}+2 n\right)^{2}}{2 n+1} \geqq \frac{1}{2 n+1} S^{2},
\end{aligned}
$$

that is,

$$
0 \geqq S^{2}-2 S\left(4 n^{2}+2 n\right)+\left(4 n^{2}+2 n\right)^{2}=\left(S-\left(4 n^{2}+2 n\right)\right)^{2} \geqq 0 .
$$

Therefore in (1.12) the equality must hold.

Q.E.D.

By using the similar technique, we see that (1.10) gives an alternate proof of one of other theorems of Okumura in [5].

Corollaly 1.6. If a Sasakian manifold $M^{2 n+1}$ is a locally symmetruc space, then $M^{2 n+1}$ is a space form.

\section{$\S 2$. Isospectral Problems of Locally $D$-Symmetric Spaces}

Special classes of Sasakian manifolds discussed in $\S 1$ are related as follows: 
$\{$ Sasakian space forms $\} \subset\{$ Locally $D$-symmetric spaces $\}$ \{C-Einstein manifolds\} \{Sasakian manifolds with $\eta$-parallel Ricci tensor\}

In this section we consider a weaker version of Theorem 0.1. Namely we prove the following theorem.

THEOREM 2.1. Let $M^{2 n+1}$ be a $2 n+1(\geqq 5)$ dimensional compact locally $D$ symmetruc C-Einstern manfold and let $M^{*}$ be a compact $C$-Einstein manifold. If $M^{2 n+1}$ and $M^{*}$ are isospectral with respect to the Laplace oprator and

$$
\int_{M} R_{\imath h}{ }^{u t} R_{u t}{ }^{k \jmath} R_{k \jmath}{ }^{i h} v_{g}=\int_{M^{*}} R^{*}{ }_{i h}{ }^{u t} R^{*}{ }_{u t}{ }^{k \jmath} R^{*}{ }_{k \jmath}{ }^{i h} \nu^{*}{ }_{g^{*}},
$$

then $M^{*}$ is a $2 n+1$ dimensional locally $D$-symmetruc space.

Proof. By regrouping each term of $a_{3}$, which is is the 4-th coefficient of the asymptotic expansion

$$
\sum_{\imath=1}^{\infty} \exp \left(-t \lambda_{\imath}\right) \sim(4 \pi t)^{-n / 2} \sum_{k=0}^{\infty} a_{k} t^{k}, \quad t \longrightarrow 0^{+}
$$

with spectrum $\left\{\lambda_{i}\right\}$ of the Laplace operator, we have

$$
a_{3}=\frac{1}{6 !} \int_{M} f v_{g}
$$

where

$$
\begin{aligned}
f= & -\frac{1}{9}\left(|\nabla R|^{2}-4|R|^{2}+16 S-32 n^{2}-16 n\right) \\
& -\frac{26}{63}\left(\mid \nabla \text { Ricci }\left.\right|^{2}-2 \mid \text { Ricci }\left.\right|^{2}+8 n S-16 n^{3}-8 n^{2}\right) \\
& -\frac{2}{3} S|R|^{2}-\frac{2}{3} S\left(\mid \text { Ricci }\left.\right|^{2}-\frac{(S-2 n)^{2}}{2 n}-4 n^{2}\right) \\
& +\frac{5}{9} S^{3}-\frac{4}{9}\left(|R|^{2}-4 S+8 n^{2}+4 n\right) \\
& -\frac{52}{63}\left(|\operatorname{Ricci}|^{2}-\frac{(S-2 n)^{2}}{2 n}-4 n^{2}\right)+\frac{208}{63} n\left(S-2 n^{2}+n\right) \\
& -\left(\frac{2}{3} S+\frac{52}{63}\right)\left(\frac{(S-2 n)^{2}}{2 n}+4 n^{2}\right)-\frac{8}{21} R_{i h}{ }^{u t} R_{u t}{ }^{k}{ }^{k} R_{k \jmath}{ }^{i h} \\
& -\frac{8}{63} R_{t j i n} R_{u}{ }^{j i n} R^{t u}-\frac{20}{63} R_{k j i n} R^{j n} R^{k \imath}-\frac{4}{7} R_{h}{ }^{\jmath} R_{\jmath}{ }^{2} R_{\imath}{ }^{h} .
\end{aligned}
$$

If we put the assumption of $C$-Einsteinness, $f$ is given by 


$$
\begin{aligned}
f= & -\frac{1}{9}\left(|\nabla R|^{2}-4|R|^{2}+16 S-32 n^{2}-16 n\right) \\
& -\frac{26}{63}\left(\mid \nabla \text { Ricci }\left.\right|^{2}-2 \mid \text { Ricci }\left.\right|^{2}+8 n S-16 n^{3}-8 n^{2}\right) \\
& -\frac{2}{3} S|R|^{2}+\frac{5}{9} S^{3}-\frac{4}{9}\left(|R|^{2}-4 S+8 n^{2}+4 n\right) \\
& +\frac{208}{63} n\left(S-2 n^{2}+n\right)-\frac{8}{21} R_{\imath h}{ }^{u t} R_{u t^{k j}} R_{k \jmath}{ }^{i n} \\
& -\frac{8}{63}\left(a|R|^{2}+4 n b\right)+\frac{20}{63}\left(a S^{2}+4 n a b\right)-\frac{4}{7}\left((a+b)^{3}+2 n a^{3}\right)
\end{aligned}
$$

where $a=\frac{S-2 n}{2 n}$ and $b=2 n-\frac{S-2 n}{2 n}$.

Now back to our particular case, first we easily see that $\operatorname{dim} M^{*}=2 n+1$ and volume of $M=$ volume of $M^{*}$. From the assumption, the scalar curvature $S$ and $S^{*}$ of $M$ and $M^{*}$ respectively are both constants. Hence $a_{1}=a_{1}^{*}$ implies that $S=S^{*}$. In general $a_{2}$ is expressed as

$$
\begin{array}{r}
a_{2}=\frac{1}{360} \int_{M}\left[2|R|^{2}-2\left(\mid \text { Ricci }\left.\right|^{2}-\frac{(S-2 n)^{2}}{2 n}-4 n^{2}\right)\right. \\
\left.+5 S^{2}-2\left(\frac{(S-2 n)^{2}}{2 n}+4 n^{2}\right)\right] v_{g} .
\end{array}
$$

Thus in our case $a_{2}=a_{2}{ }^{*}$ gives $\int_{M}|R|^{2} v_{g}=\int_{M^{*}}\left|R^{*}\right|^{2} v^{*} g^{*}$. Summing up these facts, we see that $a_{3}=a_{3}^{*}$ is simplified to

$$
\begin{aligned}
0= & \int_{M^{*}}\left[-\frac{1}{9}\left(\left|\nabla R^{*}\right|^{2}-4\left|R^{*}\right|^{2}+16 S^{*}-32 n^{2}-16 n\right)\right. \\
& \left.-\frac{26}{63}\left(\mid \nabla \text { Ricci }\left.^{*}\right|^{2}-2 \mid \text { Ricci }\left.^{*}\right|^{2}+8 n S^{*}-16 n^{3}-8 n^{2}\right)\right] v^{*}{ }_{g^{*}} .
\end{aligned}
$$

Using Propositions 1.3 and 1.4, we conclude that $M^{*}$ is a locally $D$-symmetric space.

Q.E.D.

Theorem 2.1 is a Sasakian version of Sakai's theorem [6]. In order to simplify the assumption, Sakai used the Euler characteristic. However he to sacrifice the dimension. In our case we do not know yet what assumption simplifies our theorem.

\section{$\S 3$. Isospectral Problems of Submanifolds of Sasakian Manifolds}

It is well known that the spectrum of the second variation operator $\square$ gives 
some geometric characterizations of minimal submanifolds.

Suppose $M^{n}$ is a minimal submanifold of dimension $n$ isometrically immersed in the Riemannian manifold $\bar{M}^{m}$ of dimension $m$. The second variation operator $\square$ is defined by

$$
\square W=D_{\nabla} W+\bar{R}(W)-{ }^{t} A A(W), \quad W \in \Gamma\left(T M^{\perp}\right),
$$

where $D_{\nabla}$ is the Laplace operator in the normal bundle to $M^{n}, A$ is the second fundamental tensor, $\Gamma\left(T M^{\perp}\right)$ denotes the space of smooth sections to the normal bundle and $\bar{R}: \Gamma\left(T M^{\perp}\right) \rightarrow \Gamma\left(T M^{\perp}\right)$ is the partial Ricci transformation given by

$$
\bar{R}(W)=\sum_{\imath=1}^{m}\left(R\left(e_{\imath}, W\right) e_{\imath}\right)^{N}, \quad W \in \Gamma\left(T M^{\perp}\right)
$$

for $e_{1}, \cdots, e_{m}$ an orthonormal basis of $T M,()^{N}$ denoting the normal part to $M^{n}$.

If we consider the asymptotic expansion (2.1) with spectrum $\left\{\lambda_{i}\right\}$ of the second variation operator, we have

THEOREM 3.1. (Gilkey [2])

$$
\begin{aligned}
& a_{0}(\square)=r \text { volume of } M^{n}, \\
& a_{1}(\square)=\frac{r}{6} \int_{M} S v_{g}-\int_{M}\left(\operatorname{Tr}(\bar{R})-|h|^{2}\right) v_{g},
\end{aligned}
$$

where $r=m-n=$ codimension of $M^{n}$ in $\bar{M}^{m}$ and $|h|$ is the length of the second fundamental tensor $h$.

Next, before examining our problems, we review some theory of submanifolds of Sasakian manifolds. (For more detail, see Yano and Kon [10])

Let $\bar{M}$ be a $2 m+1$ dimensional Sasakian manifold with structure tensors $(\phi, \xi, \eta)$. A submanifold $M$ in $\bar{M}$ is called a Sasakian submanifold if the characteristic vector field $\xi$ is tangent to $M$ everywhere on $M$ and $\phi X$ is tangent to $M$ for any tangent vector $X$ to $M$. Obviously, $M$ is a Sasakian manifold of dimension, say $2 n+1, n<m$, with respect to the induced structure tensors.

By checking the property of the second fundamental form, we have

Proposition 3.2. Any Sasakıan submanıfold is a mınımal submanıfold.

From the Gauss equation, we have

Proposition 3.3. Let $M$ be a Sasakian submanfold in a Sasakian space form $\bar{M}(c)$ of a constant $\phi$-sectronal curvature $c$. Then $M$ is a totally geodesic submanfold if and only if $M$ is of a constant $\phi$-sectional curvature c.

$S^{2 n+1} \subset S^{2 m+1}(n<m)$ is an example of a totally geodesic Sasakian submanifold.

If an $n$ dimensional submanifold $M$ immersed in a Sasakian manifold $\bar{M}^{2 m+1}$ satisfies $\phi T_{p} M \subset T_{p} M^{\perp}$ for each $p \in M$, where $T_{p} M^{\perp}$ is a normal space to $M$ at $p$, then $M$ is called an antr-invariant submanifold. Since the rank of $\phi$ is $2 m$, we see that $n \leqq m+1$. In the case of $n=m+1$, the characteristic vector field $\xi$ 
is necessarily tangent to $M$.

Now we go back to our problem. Let $M^{2 n+1}$ be a Sasakian submanifold in a Sasakian space form $\bar{M}^{2 m+1}(c)$ of a constant $\phi$-sectional curvature $c$. We compute the scalar curvature $S$ of $M^{2 n+1}$ by using the Gauss equation and an orthonormal basis $e_{1}, \cdots, e_{n}, \phi e_{1}, \cdots, \phi e_{n}, \xi$ of $T_{p} M$ for any $p \in M^{2 n+1}$. We have

$$
S=\frac{1}{2}(n(2 n+1)(c+3)+n(c-1))-|h|^{2}+(2 n+1)|H|^{2},
$$

where $H$ is the mean curvature vector, which is in our case 0 because of Proposition 3.2.

Next, by direct computation, we have

$$
\bar{R}(W)=-\frac{1}{4}((2 n+1)(c+3)-(c-1)) W, \quad W \in T M^{\perp} .
$$

Hence

$$
\operatorname{Tr}(\bar{R})=-\frac{r}{4}((2 n+1)(c+3)-(c-1)),
$$

where $r=(2 m+1)-(2 n+1)=$ codimension of $M^{2 n+1}$ in $\bar{M}^{2 m+1}(c)$.

Substituting (3.4) and (3.6) into (3.3), we have $a_{1}(\square)$ in our case.

$$
\begin{aligned}
a_{1}(\square)= & \int_{M}\left[\frac{r}{12}(n(2 n+1)(c+3)+n(c-1))\right. \\
& \left.+\frac{r}{4}((2 n+1)(c+3)-(c-1))+\left(1-\frac{r}{6}\right)|h|^{2}\right] v_{g} .
\end{aligned}
$$

THEOREM 3.4. Let $M$ and $M^{*}$ be compact Sasakian submanifolds in a Sasakian space form $\bar{M}(c)$. If $M$ and $M^{*}$ are isospectral with respect to $\square$ and $M$ is a totally geodesic submanifold, then $M^{*}$ is totally geodesic unless the codimension of $M^{*}$ in $\bar{M}(c)$ is 6 .

Proof. The asymptotic expansion (2.1) with spectrum of $\left\{\lambda_{i}\right\}$ shows that the codimension of $M$ and $M^{*}$ are equivalent. The equivalence of $a_{1}(\square)$ reduces to

$$
\left(1-\frac{r}{6}\right) \int_{M}|h|^{2} v_{g}=\left(1-\frac{r}{6}\right) \int_{M^{*}}\left|h^{*}\right|^{2} v^{*}{ }_{g^{*}} .
$$

Since $M$ is totally geodesic, the left side of (3.8) vanishes. Hence if $r \neq 6, M^{*}$ is also totally geodesic.

Q.E.D.

Noting Proposition 3.3, we have

COROLLARY 3.5. Let $M$ and $M^{*}$ be compact Sasakian submanifolds in a Sasakian space form $\bar{M}(c)$. If $M$ and $M^{*}$ are isospectral with respect to $\square$ and $M$ is a Sasakian space form $M(c)$, then $M^{*}$ is also a Sasakıan space form $M^{*}(c)$ unless the codimension of $M^{*}$ in $\bar{M}(c)$ is 6 . 
By applying the similar method to anti-invariant submanifolds in a Sasakian space form, we have

THEOREM 3.6. Let $M$ and $M^{*}$ compact ornentable anti-invariant minumal submanifolds in a Sasakıan space form $\bar{M}(c)$. If $M$ and $M^{*}$ are isospectral with respect to $\square$ and $M$ is totally geodesic, then $M^{*}$ is totally geodesic unless the codimension of $M^{*}$ in $\bar{M}(c)$ is 6 .

\section{$\S 4$. Isospectral Problems of Anti-Invariant Submanifolds of Complex Manifolds}

Donnally [1] proved an isospectral problem of Kaehler submanifolds in a complex space form by using the second variation operator.

Anti-invariant submanifolds of complex manifolds constitute another interesting class of submanifolds in complex manifolds.

By slightly modifying Donnally's proof, we easily prove

THEOREM 4.1. Let $M$ and $M^{*}$ be compact orientable anti-invariant minimal submanifolds in a complex space form $\bar{M}(c)$. If $M$ and $M^{*}$ are isospectral with respect to $\square$ and $M$ is totally geodesic, then $M^{*}$ is totally geodesic unless the codimension of $M^{*}$ in $\bar{M}(c)$ is 6 .

\section{$\S 5$. Isospectral Problems of Quaternion Kaehler Manifolds}

First we review the definition and some properties of quaternion Kaehler manifolds. (For more detail, see Ishihara [3])

A $4 n$ dimensional Riemannian manifold $M^{4 n}$ is said to be a quaternion Kaehler manfold if and only if the holonomy group if a subgroup of $S p(n) \cdot S p(1)$ $=S p(n) \otimes S p(1) /\{ \pm 1\}$, where $S p(n)$ is the real representation of the symplectic unitary group acting on $C^{2 n}$. In other words, there exists a 3 dimensional vector bundle $V$ consisting of tensors of type $(1,1)$ over $M^{4 n}$ such that in any coordinate neighborhood $U$ of $M^{4 n}$, there is a local basis $\{F, G, H\}$ of $V$ satisfying

$$
\begin{gathered}
F^{2}=-I, \quad G^{2}=-I, \quad H^{2}=-I, \\
G H=-H G=F, \quad H F=-F H=G, \quad F G=-G F=H,
\end{gathered}
$$

and

$$
\begin{aligned}
& \nabla_{X} F=r(X) G-q(X) H, \\
& \nabla_{X} G=-r(X) F \quad+p(X) H, \\
& \nabla_{X} H=q(X) F-p(X) G,
\end{aligned}
$$

for the Riemannian connection $\nabla$ of $M^{4 n}$, where $X$ is any vector field on $M^{4 n}$, $p, q$ and $r$ are certain local 1 -forms defined in $U .\{F, G, H\}$ is called a canonical 
local basis of the bundle $V$ over $U$. Suppose $\left\{F^{\prime}, G^{\prime}, H^{\prime}\right\}$ is a canonical local basis over $U^{\prime}$, where $U \cap U^{\prime}=\emptyset$, we have

$$
\begin{aligned}
& F^{\prime}=s_{11} F+s_{12} G+s_{13} H, \\
& G^{\prime}=s_{21} F+s_{22} G+s_{23} H, \\
& H^{\prime}=s_{31} F+s_{32} G+s_{33} H
\end{aligned}
$$

with functions $s_{j i}$ in $U \cap U^{\prime}$. A $(3,3)$ matrix $\left(s_{j i}\right)$ belongs to the proper orthogonal group $S O(3)$ of dimension 3. Thus any quaternion Kaehler manifold is orientable.

We take a point $p$ in a quaternion Kaehler manifold $M^{4 n}$ of dimension $4 n$ and a tangent vector $X$ of $M^{4 n}$ at $p$. Then the 4 dimensional subspace $Q(X)$ of $T_{p} M^{4 n}$ defined by

$$
Q(X)=\left\{Y \in T_{p} M^{4 n}: Y=a X+b F X+c G X+d H X, a, b, c, d \in \boldsymbol{R}\right\}
$$

is called the $Q$-section determined by $X$. For any $Y, Z \in Q(X)$, if the sectional curvature $\sigma(Y, Z)$ is a constant $\rho(X), \rho(X)$ is called the Q-sectional curvature with respect to $X$ at $p$. Moreover if we suppose that $\rho(X)$ is a constant $c=c(p)$ independent of $X$ at each point of $p$, then the quaternion Kaehler manifold $M^{4 n}$ is said to have a constant $Q$-sectional curvature $c(p)$.

The following two theorems are well known.

THEOREM 5.1. A quaternion Kaehler manifold of dimension $\geqq 8$ is of a constant $Q$-sectional curvature $c=c(p)$ if and only if its curvature tensor has the components of the form

$$
\begin{aligned}
R_{k j i h}= & \frac{1}{4} c\left(g_{j \imath} g_{k h}-g_{k i} g_{j h}+F_{j i} F_{k h}-F_{k \imath} F_{j h}-2 F_{k \jmath} F_{i n}\right. \\
& \left.+G_{j i} G_{k h}-G_{k i} G_{j h}-2 G_{k j} G_{i h}+H_{j i} H_{k h}-H_{k \imath} H_{\jmath h}-2 H_{k \jmath} H_{\imath h}\right) .
\end{aligned}
$$

THEOREM 5.2. For a quaternon Kaehler manifold of a constant Q-sectional curvature $c=c(p), p \in M$, the function $c(p)$ is constant in $M$ if $\operatorname{dim} M \geqq 8$.

A quaternion Kaehler manifold $M^{4 n}$ with a constant $Q$-sectional curvature $c$ is called a quaternion space form $M^{4 n}(c)$. The Ricci tensor $R_{\jmath i}$ and the scalar curvature $S$ of a quaternion space form are given by

$$
\begin{aligned}
& R_{j i}=\frac{1}{2} c(2 n-5) g_{j i}, \\
& S=2 n c(2 n-5) .
\end{aligned}
$$

The quaternion projective space is an example of a quaternion space form of $c=4$.

Let $M^{4 n}$ be a quaternion Kaehler manifold of $\operatorname{dim} M^{4 n} \geqq 8$. We now define a tensor $D$ in $U$ which may correspond to the Bochoner curvature tensor in Kaehler 
manifolds. The components of $D$ in $U$ are given by

$$
\begin{aligned}
D_{k j i h}= & R_{k j i h}-\frac{1}{4(n+4)}\left(R_{j i} g_{k h}-R_{k i} g_{j h}+g_{j i} R_{k h}-g_{k \imath} R_{j h}\right. \\
& +R_{i t} F_{\jmath}{ }^{t} F_{k h}-R_{i t} F_{k}{ }^{t} F_{j h}-2 R_{j t} F_{k}{ }^{t} F_{i h} \\
& +F_{j i} R_{h t} F_{k}{ }^{t}-F_{k \imath} R_{h t} F_{\jmath}{ }^{t}-2 F_{k j} R_{h t} F_{\imath}{ }^{t} \\
& +R_{i t} G_{\jmath}{ }^{t} G_{k h}-R_{i t} G_{k}{ }^{t} G_{j h}-2 R_{j t} G_{k}{ }^{t} G_{i h} \\
& +G_{j i} R_{h t} G_{k}{ }^{t}-G_{k \imath} R_{h t} G_{\jmath}{ }^{t}-2 G_{k j} R_{h t} G_{\imath}{ }^{t} \\
& +R_{i t} H_{\jmath}{ }^{t} H_{k h}-R_{i t} H_{k}{ }^{t} H_{j h}-2 R_{j t} H_{k}{ }^{t} H_{i h} \\
& \left.+H_{j i} R_{h t} H_{k}{ }^{t}-H_{k \imath} R_{h t} H_{\jmath}{ }^{t}-2 H_{k j} R_{h t} H_{\imath}{ }^{t}\right) \\
& +\frac{S}{16(n+2)(n+4)}\left(g_{j i} g_{k h}-g_{k i} g_{j h}+F_{j i} F_{k h}\right. \\
& -F_{k i} F_{j h}-2 F_{k j} F_{i h}+G_{j i} G_{k h}-G_{k i} G_{j h} \\
& \left.-2 G_{k j} G_{i h}+H_{j i} H_{k h}-H_{k i} H_{j h}-2 H_{k j} H_{i h}\right) .
\end{aligned}
$$

Because of (5.3), we see that $D$ is actually a global tensor on $M^{4 n}$. It is easily checked that if $D=0$ and $M^{4 n}$ is Einstein, then $M^{4 n}$ is a quaternion space form. On the other hand if $M^{4 n}$ is a quaternion space form, then $M^{4 n}$ is Einstein and $D=0$. However we have

THEOREM 5.3. Any quaternon Kaehler manifold of dimension $\geqq 8$ is an Einstern manifold.

Hence $D$ is reduced to

$$
\begin{aligned}
D_{k \imath j h}= & R_{k j i h}-\frac{S}{16 n(n+2)}\left(g_{j i} g_{k h}-g_{k i} g_{j h}+F_{j i} F_{k h}-F_{k i} F_{j h}-2 F_{k j} F_{i n}\right. \\
& \left.+G_{j i} G_{k h}-G_{k i} G_{j h}-2 G_{k j} G_{i n}+H_{j i} H_{k h}-H_{k i} H_{j h}-2 H_{k j} H_{i h}\right) .
\end{aligned}
$$

By using the curvature properties of quaternion Kaehler manifolds found in Ishihara [3], we see that

$$
|D|^{2}=|R|^{2}-\frac{5 n+1}{4 n(n+2)^{2}} S^{2}
$$

Therefore we have

THEOREM 5.4. Let $M^{4 n}$ be a quaternion Kaehler manifold of dimension $\geqq 8$. An inequality

$$
|R|^{2} \geqq \frac{5 n+1}{4 n(n+2)^{2}} S^{2}
$$


holds on $M^{4 n}$. Equality holds if and only of $M^{4 n}$ is a quaternon space form $M^{4 n}(c)$, where $c=\frac{S}{2 n(2 n-5)}$.

Now we consider an isospectral problem of quaternion Kaehler manifolds by using the asymptotic expansion (2.1) with the spectrum of the Laplace operator.

THEOREM 5.5. Let $\left(M^{4 n}(c), g\right)$ be a compact quaternon space form of a constant $Q$-sectional curvature $c$ of dimension $4 n \geqq 8$, and let $\left(M^{*}, g^{*}\right)$ be a compact quaternion Kaehler manifold. If $\operatorname{Spec}\left(M^{4 n}(c), g\right)=\operatorname{Spec}\left(M^{*}, g^{*}\right)$, then $M^{*}$ is a quaternion space form of constant $Q$-sectional curvature $c^{*}=c$ of dimension $4 n$.

Proof. From (2.1) we easily see $\operatorname{dim} M^{*}=4 n$ and the volume of $M^{4 n}(c)=$ volume of $M^{*}$. Since both $M^{4 n}(c)$ and $M^{*}$ are Einstein manifolds by Theorem 5.3, the scalar curvature $S$ and $S^{*}$ are constants. Hence the condition $a_{1}=a_{1}{ }^{*} \mathrm{im}$ plies that $S=S^{*}$. Using the Einsteinness we can express $a_{2}$ as

$$
\begin{aligned}
a_{2} & =\frac{1}{360} \int_{M}\left(2|R|^{2}-2 \mid \text { Ricci }\left.\right|^{2}+5 S^{2}\right) v_{g} \\
& =\frac{1}{360} \int_{M}\left(2|R|^{2}+\left(5-\frac{1}{2 n}\right) S^{2}\right) v_{g} \\
& =\frac{1}{360} \int_{M} 2\left(|R|^{2}-\frac{5 n+1}{4 n(n+2)^{2}} S^{2}\right) v_{g}+\frac{1}{360} \int_{M} \frac{20 n^{3}+78 n^{2}+77 n-7}{4 n(n+2)^{2}} S^{2} v_{g} .
\end{aligned}
$$

Therefore $a_{2}=a_{2} *$ in our case implies

$$
\begin{aligned}
\frac{20 n^{3}+78 n^{2}+77 n-7}{4 n(n+2)^{2}} S^{2} \int_{M} v_{g}= & \int_{M^{*}} 2\left(\left|R^{*}\right|^{2}-\frac{5 n+1}{4 n(n+2)^{2}} S^{* 2}\right) v^{*} g^{*} \\
& +\frac{20 n^{3}+78 n^{2}+77 n-7}{4 n(n+2)^{2}} S^{* 2} \int_{M^{*}} v^{*} g^{*}
\end{aligned}
$$

Because of Theorem 5.4, we see that $M^{*}$ is a quaternion space form, and (5.6) shows $c^{*}=c$.

Q.E. D.

\section{BIBLIOGRAPHY}

[1] H. Donnelly, Spectral invariants of the second variation operator, Illinois J. Math. 121 (1977), 185-189.

[2] P.B. Gilkey, The spectral geometry of real and complex manifolds, Proc. Sympos. Pure Math., Vol 27 part 2, Amer. Math. Soc., Providence, R.I., 1975, 265-280.

[3] S. Ishinara, Quaternion Kaehler manifolds, J. Differential Geometry 9 (1974), 483-500.

[4] M. Kos, Invariant submanifolds in Sasakian manifolds, Math. Ann. 219 (1976), 227-290.

[5] M. OKtiltra, Some remarks on space with a certain contact structure, Tôhoku 
Math. J. 14 (1962), 135-145.

[6] T. SAKAI, On eigenvalues of Laplacian and curvature of Riemannian manifold, Tôhoku Math. J. 23 (1971), 589-603.

[7] Y. ShibuYA, The Spectrum of Sasakian manifolds, Kodai Math. J. 3(1980), 197211.

[8] Y. SHibuYA, Isospectral problems of Sasakian manifolds and their submanifolds, Tokyo Institute of Technology, 1980.

[9] T. Takahashi, Sasakian $\phi$-symmetric spaces, Tôhoku Math. J. 29 (1977), 91113.

[10] K. Yano \& M. Kon, Anti-Invariant Submanifolds, Lecture Notes in pure and appl. Math., Vol. 21, Marcel Dekker Inc., New York and Basel, 1976.

Department of Mathematics,

Tokyo Institute of Technohogy,

Oh-okayama, Meguro, 152, Tokyo, Japan 